\title{
Querying in Business Process Modeling
}

\author{
Ivan Markovic ${ }^{1}$, Alessandro Costa Pereira ${ }^{1}$, David de Francisco ${ }^{2}$, \\ and Henar Muñoz ${ }^{2}$ \\ ${ }^{1}$ SAP Research, Karlsruhe, Germany \\ \{ivan.markovic, alessandro.costa.pereira\}@sap.com \\ 2 Telefónica Research and Development, Valladolid, Spain \\ \{ddf268,hmf268\}@tid.es
}

\begin{abstract}
To respond quickly to changing market requirements, businesses need to increase the level of agility in all phases of the business process engineering chain. Business process (BP) modeling is the first and most important phase in this chain. Designing a new and redesigning an existing process model is a highly complex, time consuming and error prone task. In this work, we contribute to BP modeling by i) analyzing the usage scenarios and identifying the types of queries which facilitate the design and increase quality of newly created BP models and ii) devising an approach to support querying in BP modeling.
\end{abstract}

\section{Introduction}

In the modern world, businesses constantly strive to reinvent and differentiate themselves under continuous pressures of regulatory and technological change, as well as the increasingly time to market requirements. One of the main obstacles for these changes to be agile is the lack of support when incorporating new business requirements into existing information systems as priorities and perspectives change.

Business process (BP) modeling is the first and most important phase in the business process engineering chain. BP models are created by business analysts with an objective to capture business requirements, enable a better understanding of business processes, facilitate communication between business analysts and IT experts, identify process improvement options and serve as a basis for derivation of executable business processes. Designing a new process model is a highly complex, time consuming and error prone task. This is because BP modeling involves several sources of information, models are dynamic and frequently redesigned to adapt changes, and BP models are often shared by several departments within a company or even between different companies.

In order to simplify BP modeling, models must be highly reusable, favoring process flexibility and minimizing designs made from scratch. Reusing implies querying the process repository to find suitable previous work that can be the base for a new design, and this can only be made by describing relevant aspects of a BP model that will help to retrieve the expected results. The definition of a business process model comprises two different aspects: the dynamic (behavioral) 
aspect and the static view of a process. While the dynamic aspect captures control and dataflow of a process model, the static view defines the context of the process comprised of a business domain, business function, roles and resources [1. In the following we define some of the concepts used throughout this paper, namely business goals, BP patterns and modeling guidelines.

From the functional perspective, processes achieve some business goals according to a certain business function. A business goal can be defined as an objective or milestone within the process which is meaningful enough from a business perspective, and thus does not deal with execution parameters. A business function defines what a process intends to achieve from a business point of view. The definition of the process through subprocesses, process fragments, tasks and services defines how these objectives are achieved. Business goals can be categorized in the functional map of a company.

BP modeling is implicitly based on abstract models that we will define as business process patterns (BP patterns). BP patterns are abstract BPs, which are not fully defined in terms of concrete tasks and services, and thus can not be executed. These patterns capture how the business analyst defines a first draft of a process, making emphasis on the business goals, and leaving the concrete definition of processes to a further step. They represent a solution to a well known problem, and a base to enable the extension to a concrete problem (as software design patterns [2] do), providing best modeling practices.

Any BP model has to comply with a set of modeling guidelines defined by business managers within a company. Guidelines are concrete policies which orthogonally apply to all business processes of the company, derived from the general strategies of the company such as getting as many customers as possible or reducing costs no matter if the quality of the service decreases. Note that we will use the term BP artifacts when we refer to BP patterns, models, fragments and modeling guidelines.

In our previous work [1] we presented a rich formal model which integrates different workflow perspectives for a comprehensive process model description. Here we take a step further and contribute to semantic BP modeling [3] by i) analyzing the usage scenarios and identifying the types of queries which facilitate the design and increase quality of newly created BP models and ii) devising an approach to support querying in BP modeling.

The remainder of the paper is structured as follows. In Section 2 we present requirements analysis for querying in BP modeling. Section 3 describes our devised approach for querying and provides some illustrative examples. Section 4 discusses related work. We conclude and give an outlook on future research in Section 5 .

\section{Requirements Analysis}

As a first step in the analysis of requirements we describe a number of sample usage scenarios for our approach. Based on the proposed usage scenarios we derive a set of requirements for the devised approach. 


\section{$2.1 \quad$ Usage Scenarios}

In Figure 1 we present an overview of all usage scenarios. The FMC1 diagram shows the interaction between the analyst and the querying and repository components.

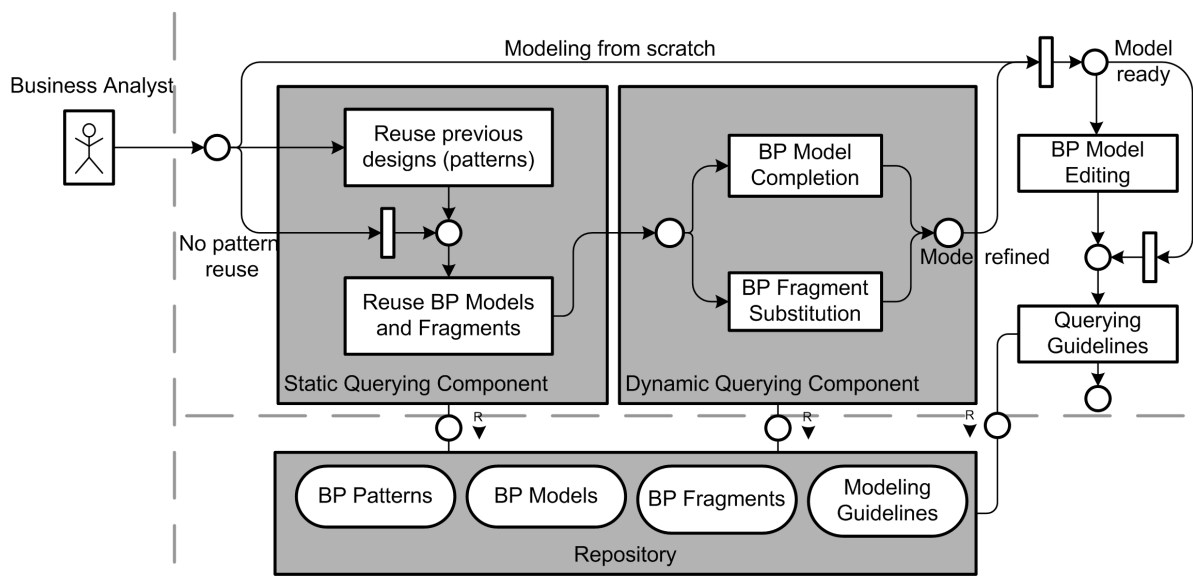

Fig. 1. Business Process Modeling Flow

\section{- Scenario 1: Goal-based process artifact search}

This scenario covers the goal-based search where the user formulates his request (goal) consisting of a set of criteria using a query template. It is covered by the Static Querying Component shown in Figure 1. The user can specify constraints on static (function, domain, role, resource) aspects of the $\mathrm{BP}$ artifact description. This request is then matched to the BP artifact descriptions from the repository and a ranked list of artifacts matching the specified goal is presented to the user. Described scenario allows business experts to quickly and expressively query the process artifact repository of an organization. Some example user queries include: "Give me all billing processes" or "Do we have a cost approval process for items below €300?" 3]. We distinguish between three cases for this scenario:

- Case 1: Design-level search Here the user queries the repository for existing high-level generic process designs - business process patterns. Patterns are queried based on their context information (business function, domain). Using these patterns as a starting point, the user can refine them to his specific needs.

- Case 2: Process model/fragment search In this case, the user queries for existing process models and fragments which he can reuse as-is or modify. This case occurs when the final model is very similar to the existing models/fragments, therefore the level of reuse is more fine-grained.

\footnotetext{
${ }^{1}$ Fundamental Modeling Concepts, http://www.f-m-c.org
} 
- Case 3: Process model refinement This scenario enables the users to create underspecified process models and let the modeling tool suggest a refinement of these models for them. We see this as a significant improvement comparing to current modeling techniques where users need to model everything in detail.

After performing static queries, we can use the Dynamic Querying Component (see Figure 1) to specify graphical queries. We consider this way of querying to be more intuitive to the user. There are two scenarios which we consider for graphical querying, process model autocompletion and process fragment substitution.

- Scenario 2: Process model autocompletion

We distinguish between two cases of autocompletion for this scenario:

- Case 1: Here we assume that the user has already modeled a part of the process and wants to find process fragments that can complete what he started to model. The modeled part of the process is matched against the process fragment descriptions from the repository and the user can select the most suitable fragment from the resulting list to include it in his design.

- Case 2: After the user selects process fragments that refine the underspecified parts of their model (Scenario 1), the system can suggest how to connect them to form the end to end process model. The input and output documents of process fragments and their context information (business domain, business function) can be used as criteria for suggesting their inter-connections.

- Scenario 3: Process fragment substitution

This scenario supports the replacement of a selected process fragment with the one that e.g. corresponds to the new process redesign goals. The newly discovered fragment has to be equivalent to the old one in terms of behavior so that the remaining process parts can stay unchanged. This functionality enables the user to easily find relevant process fragment candidates for substitution.

\section{- Scenario 4: Querying modeling guidelines}

This scenario covers the final validation of a BP model against the business guidelines defined according to the company strategy. As the user can modify the reused artifacts at any time, querying for guidelines is done after the process model is designed, as shown in Figure 11. Queries involved in this scenario retrieve all modeling guidelines (both mandatory and conditional) which match context annotations of the model being checked. Information retrieved includes a textual explanation of the modeling guideline as well as a control definition (subprocess) which explains how this guideline should be included or taken into account in the model being checked.

\subsection{Requirements}

In this section we provide a list of requirements, based on described scenarios, that an approach for querying in business process modeling should fulfill. 
- Req. 1: Rich process description The process model needs to be formally described to enable automatic matchmaking of user requests (goals) against process descriptions. In order to support the user to expressively search the process repository, we need a process description rich enough to capture both dynamic and static characteristics of a process model. For describing the dynamic (behavioral) characteristics we use process algebra, the $\pi$-calculus, and for representing static characteristics we developed a set of ontologies. In our previous work [1] we discuss in detail how we met this requirement.

- Req. 2: Intuitive user request specification The user must be provided with a user-friendly query interface for specifying his requests. The user must be able to query for processes both on the static and dynamic aspects of their description.

- Req. 3: Query language There needs to be a query language with expressive power that is sufficient to formally describe the user requests. Note that the user request can be in the form of a query template, but it can also be a part of a process for autocompletion or a process fragment for substitution.

- Req. 4: Querying mechanism There needs to be a mechanism that will perform expressive matchmaking of user requests against process descriptions. The algorithm should take a user request as an input and return a ranked list of $\mathrm{BP}$ artifacts that match the request.

\section{An Approach for Querying in Business Process Modeling}

In this section, we describe our approach to support querying in BP modeling. With the following approach, in this work we address the requirements Req. 3 and Req. 4 described in Section 2.2. To reduce the level of complexity, we have divided this task in two subtasks - static and dynamic (behavioral) querying. Figure 1 depicts the querying and repository components and their interactions. The querying mechanism is operating on the Business Process Ontology (BPO), presented in [1].

\subsection{Static Querying}

First subtask investigates simple (static) querying, i.e. the user can specify requests on static parts of a process description. Here we use WSML logical expressions [4] as a query language. The second subtask investigates graphical (behavioral) querying, where the user can specify requirements on dynamic parts of a process description. This corresponds to autocompletion and substitution usage scenarios described in 2.1 .

In the following, we provide some examples on static querying.

Example 1: Querying for design reuse. An example of a service provision Business Process Pattern by using a BPMN notation is depicted in Figure 2 In this example, a simple flow is defined between several abstract subprocesses, which will be define in further modeling steps. Annotations (denoted by circles) 
represent the business goals that mark the different milestones of the end-to-end process. By using it, the business analyst can annotate each of the phases of the pattern in order to find suitable processes for the implementation one.

A query example for business pattern: "Give me all business patterns related to Fulfillment Business Function and Client and Product Business Domain where Business Goals involved are profileObtained and serviceActivated" translates to logical expressions for querying process descriptions as follows:

Listing 1.1. Static query in WSML Logical Expression

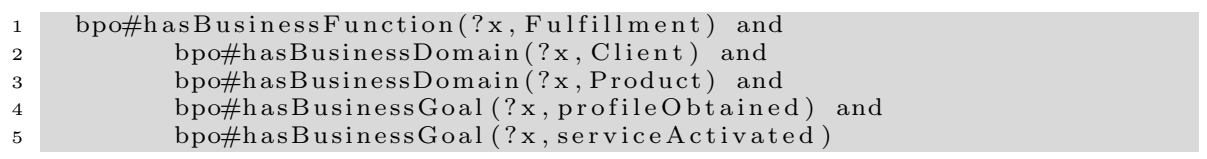

The procedure will find only processes "?x" annotated with all required characteristics. If necessary, it is possible to substitute the operator "AND" by "OR" operators, for relaxing the query.

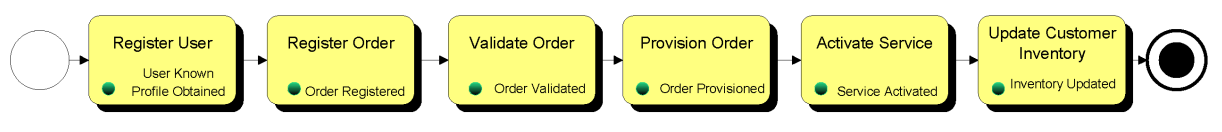

Fig. 2. Service Provision Business Pattern

The representation of pattern depicted in Figure 2 is moved to our formalism, and it is partly presented in Listing [1.2 Each procedure of the pattern is represented as a simple task.

Listing 1.2. A Pattern in BPO

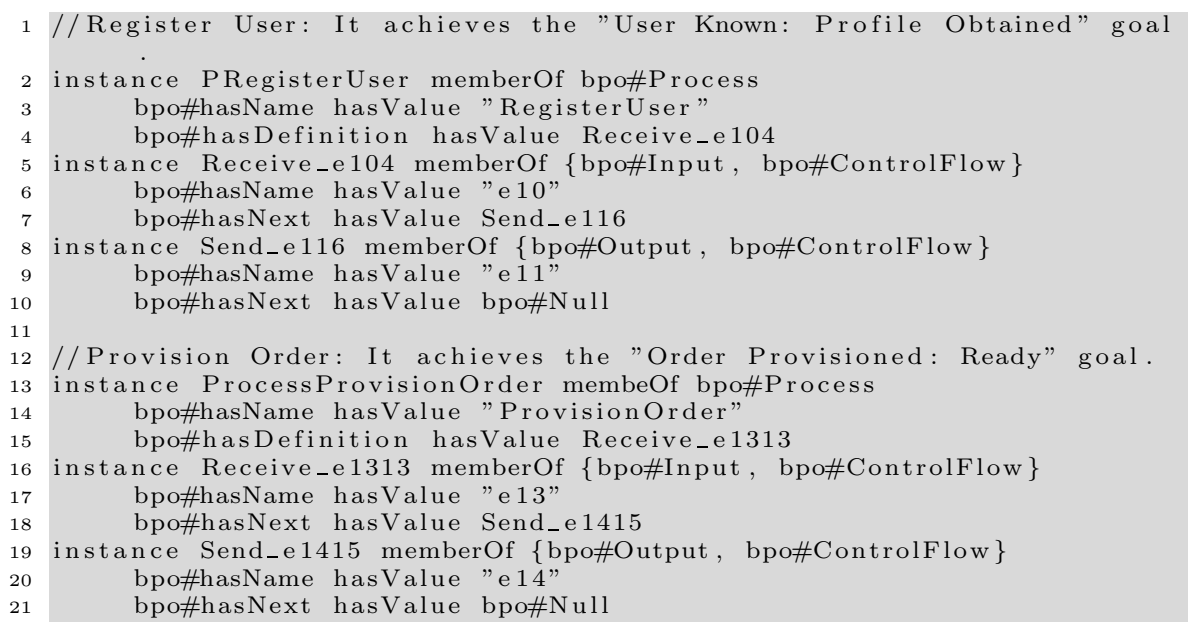

There exist a relation between each event and the respective business goal (as well, relations with domains, goals and roles), and this annotations are shown in Listing 1.3. 
Listing 1.3. Business Function Annotations

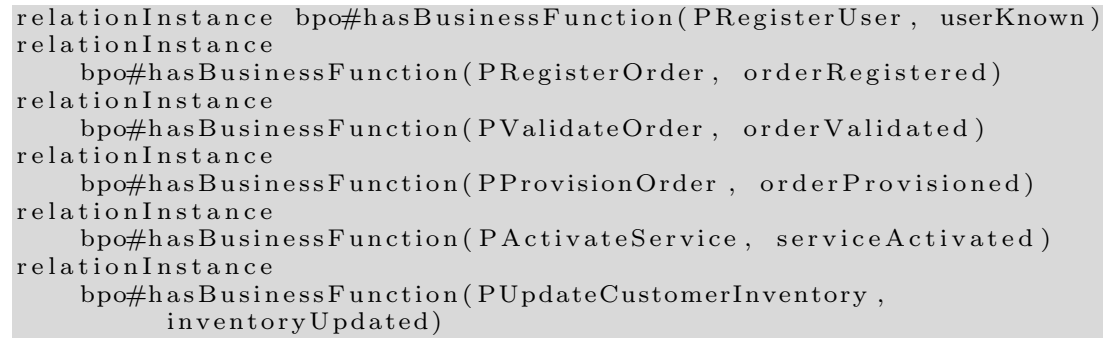

Example 2: Process model refinement. Now that the business analyst has the model presented in Figure 2 in his modeling tool, he can continue modeling. He is in the refinement phase, where he needs to find implementation fragments for each abstract task. The action he has to perform is to select the task, for instance Provision Order, for refinement. The modeling tool will look for the models or fragments annotated by the goal Order Provisioned, enabling the user to embed the Digital Asset Provision: Download Process in the model, as shown in Figure 3 .

Example 3: Querying modeling guidelines. An example for guidelines is the Parental Control for Digital Asset Management (DAM) Product, which implies that all processes that satisfying a set of characteristics (process context) must implement subprocesses which achieve the business goal Minors Monitoring. Concretely the context can be constituted by specifying: Business Function (Process Area: Manage Contact) and Business Domain (Product Area: DAM, Client Area: Minor and Localization Area: Spain).

Query example: "Give me all modeling guidelines for Digital Asset Management domain for minors where Business Goal associated belongs to Fulfillment" can be specified in the similar way to the query in Listing 1.1 .

\subsection{Dynamic Querying}

The concept of bisimilarity stands for the behavioral equivalence between two processes definition, i.e., where both processes have similar execution flow. The strong bisimilarity is the most fundamental equivalence, which evolves to the most including congruence.

In the generic case, bisimulation is a symmetric binary relation $\Re$ between two agents, in which if agent $P$ can do an action, agent $Q$ can do the same. The definition of structural congruence (operator ' $\equiv$ ') indicates that processes have the same behavior [5. Two processes are structural congruent if they behave the same, having same internal structure, however, allowing ordering reallocation. Parallelism is also considered (e.g. $P|(Q \mid R) \equiv(P \mid Q)| R$ or $P \mid 0 \equiv P)$.

We use the term strict congruent for the case where two process definitions have the same structure. Weak bisimulation is more relaxing. The idea is to make the transitions $\tau$ unobservable, by creating a transition $(\Rightarrow)$ meaning $(\stackrel{\tau}{\rightarrow})^{*}$. 
Our current approach is supporting a simple strict congruence algorithm, which can already handle complex process definitions, however, complex business processes rarely have exact the same definition. This way, such algorithm is able to find fragments which we can call Tasks definition.

The algorithm is based on the definition of bisimulation from [5. It can be represented in the simplified pseudo-code of Listing 1.4 and 1.5 .

\section{Listing 1.4. Strict Congruence Algorithm}

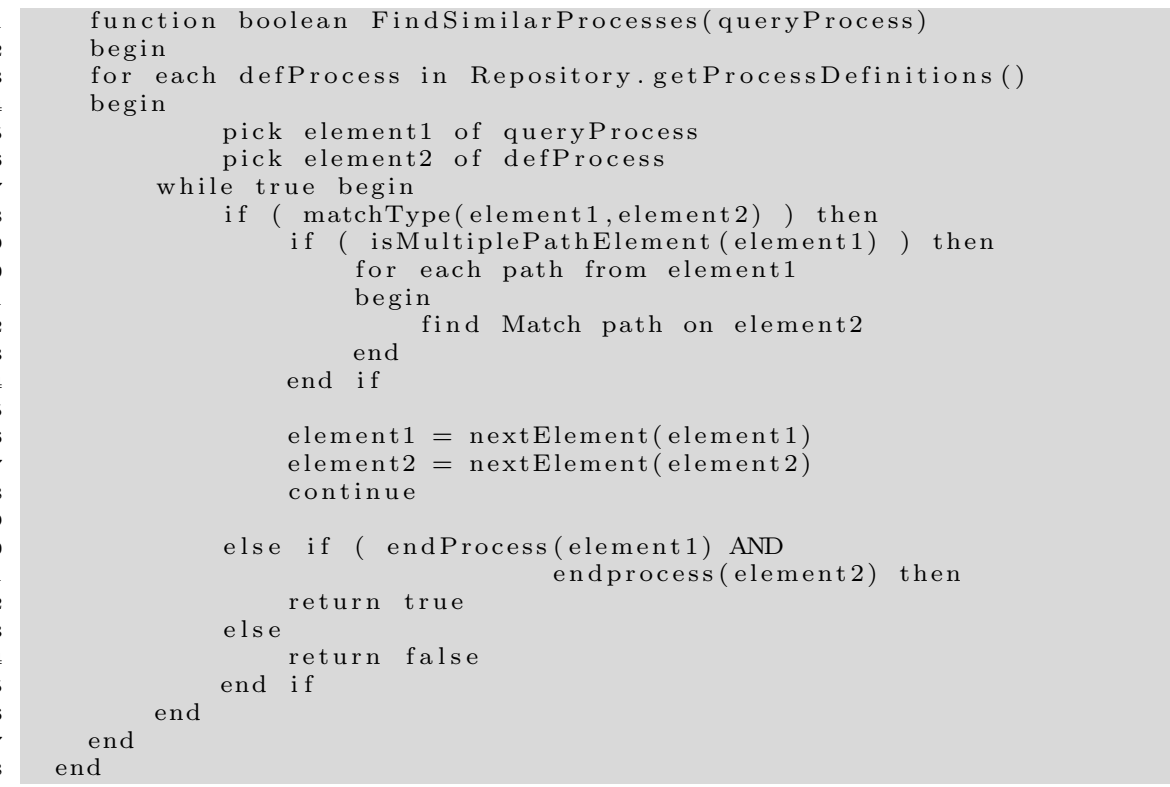

Listing 1.5presents the algorithm part of asserting types of two elements. It is characterized by the fact that names of channels should match in both processes.

Listing 1.5. Type Assertion Algorithm

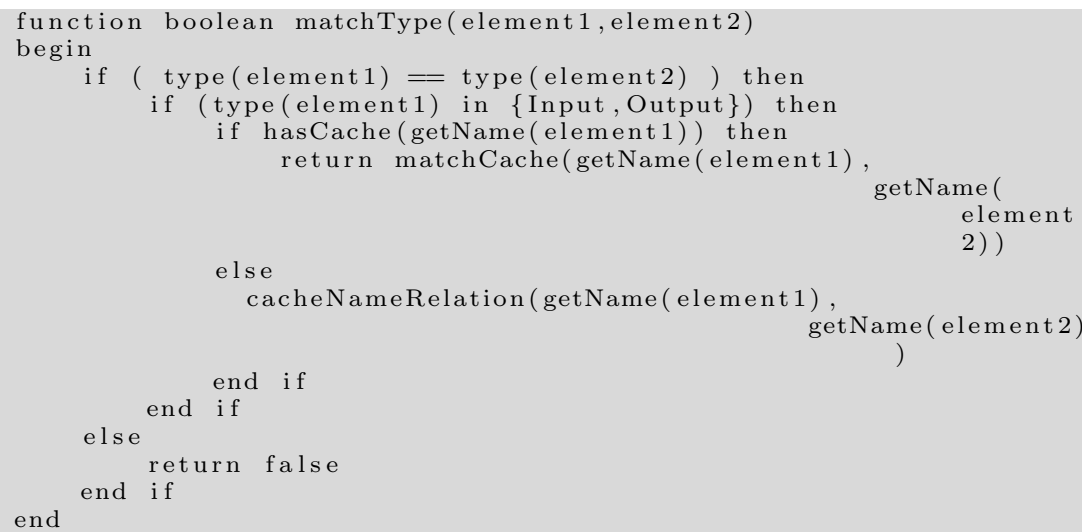


Using behavioral queries, the business analyst can query the repository in a more intuitive way, i.e. in the same way he models the processes. In the following, we present two example cases for behavioral querying which our current prototype supports.

Example 1: Process fragment substitution. Download Process (Figure 3) from DAM domain is a candidate to implement Provision Order step from Figure 2 for Digital Asset Provision Process. However, in case that the business analyst wants to change the service modality from downloading to streaming, he needs a substitution functionality, where he can use a graphical query, allowing to change the concrete process implementation by another similar. The query is specified by selecting all subprocess' elements of the Download Process. This query is submitted as a simple process model with annotations, and the dynamic querying component responds with processes (a) and (b) illustrated in Figure 4 .

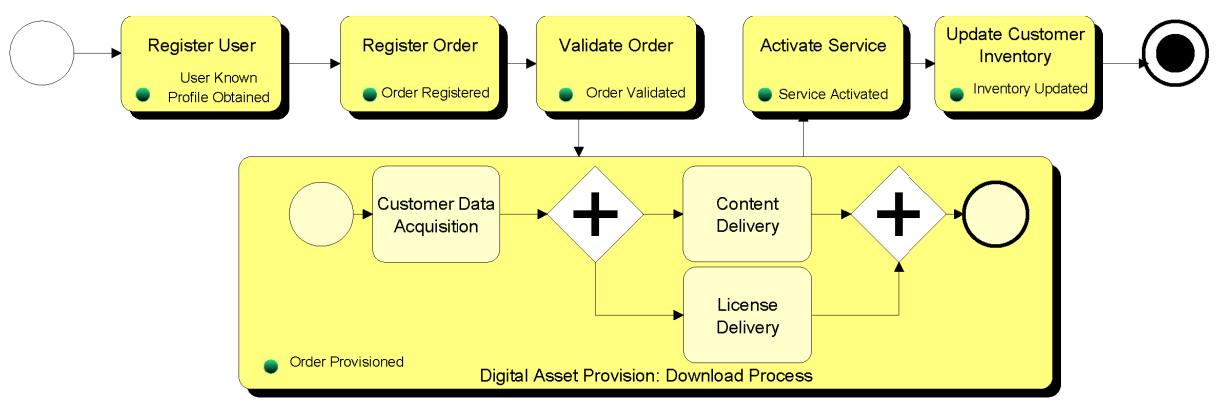

Fig. 3. Digital Asset Provision: Download Process

The subprocess substituting the pattern Provision Order has overall definition given by the model in BPO presented in Listing 1.6. Definition of the streaming subprocess has very similar structure, reason for the positive match using the algorithm mentioned in Section 3.2 .

Listing 1.6. Downloading Asset Sub-process

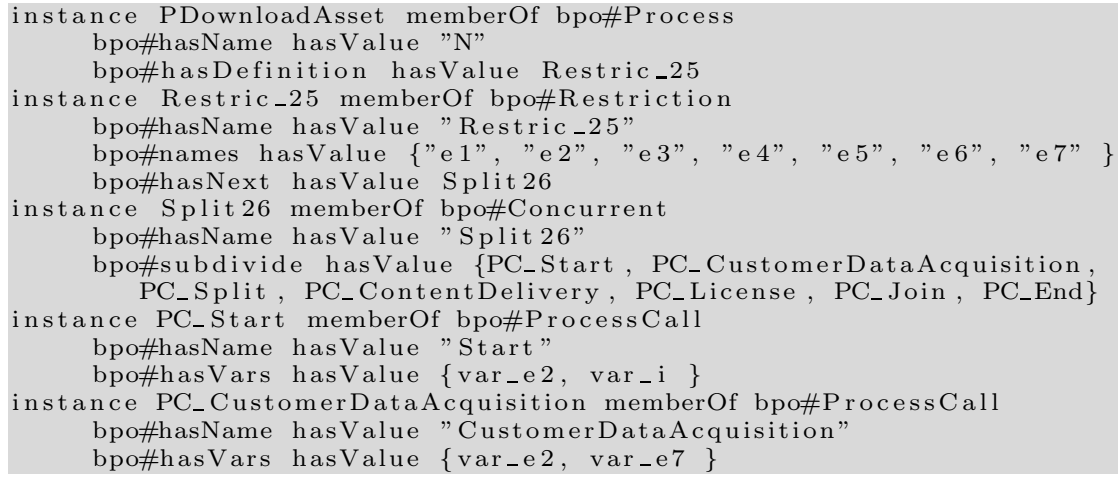




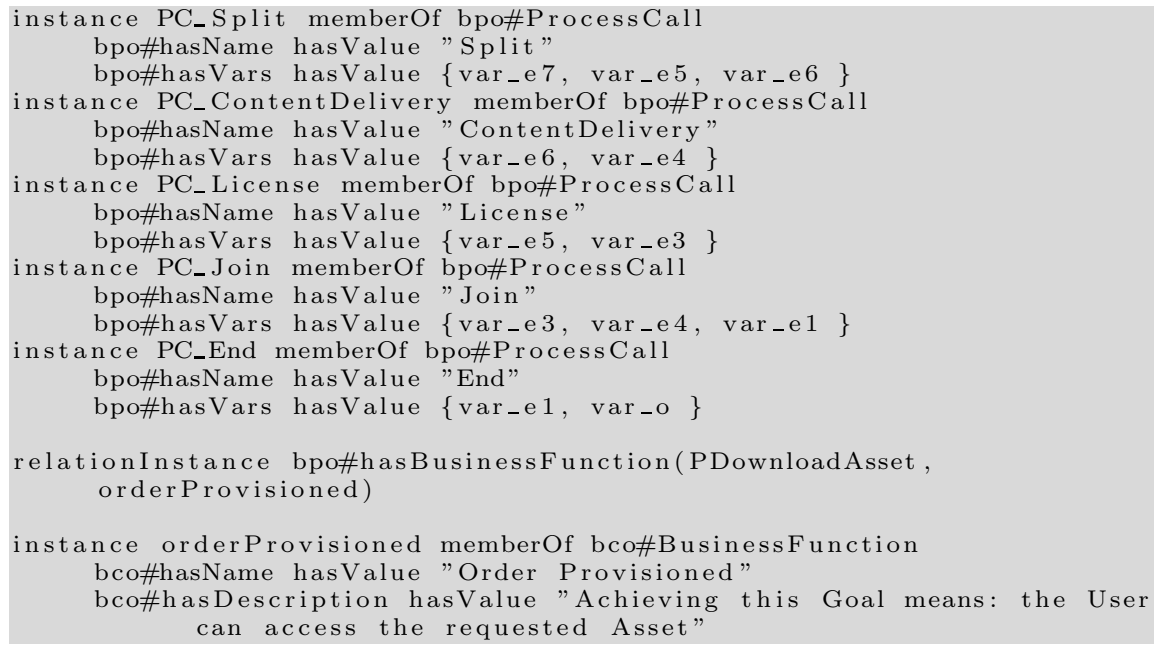

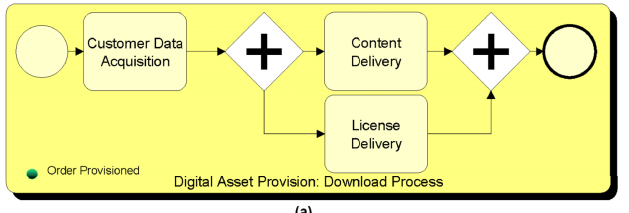

(a)

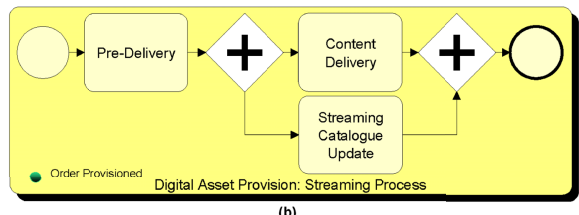

(b)

Fig. 4. Digital Asset Provision: Substitution Candidates

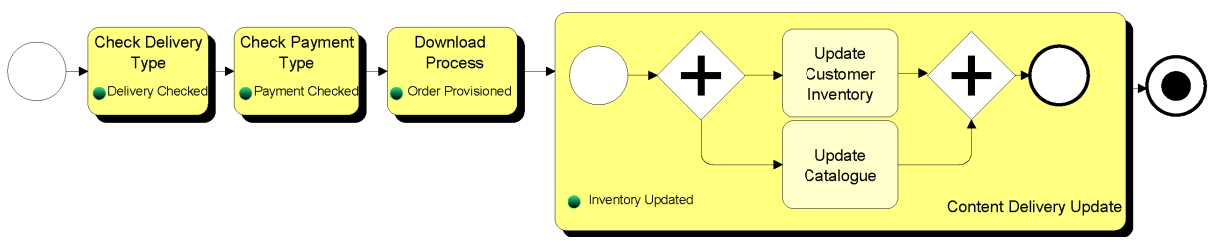

Fig. 5. Product Provision Business Process

Example 2: Process model autocompletion. Both Download and Streaming processes are always followed by "Contents Delivery Update" fragment. By performing queries, the dynamic querying component can autocomplete the end to end process to create the Product Provision Process shown in Figure 5, where Download Process belongs to.

The autocompletion uses an extension of the structural congruence algorithm, which relaxes the stop condition while searching for remaining process part in the repository. The matchmaking starts comparing subsequent tasks in the model definition against process artifacts in the repository. If the algorithm detects similarity in the initial set of tasks, it recommends the user to complete the model by using the remaining part of the most similar artifact found. 
In the process represented in Figure 5, the user started a new process from scratch, he has annotated the initial three tasks with respective goals, and has submitted it as a query for autocompletion. The dinamic querying component recognizes a pattern in the repository, where the goal Inventory Updated should follow the tasks achieving the goals Delivery Checked, Payment Checked and Order Provisioned. If the user accepts the recommendation, he can consequently look for a process fragment implementing the goal.

\section{Related Work}

The significance of querying business processes has been acknowledged by BPML2 who launched a Business Process Query Language (BPQL) initiative [6]. However, no standard specification has been published yet.

In [7, the authors present a query language for querying business processes, BP-QL. The query language is designed based on the BPEL3 standard and thus focuses on querying executable processes. Our work focuses on the reuse of higher level business knowledge, i.e. BP knowledge artifacts. In addition, our query specification language is more expressive in that apart from constraints on data and control flow, the user can describe the context of the models he wants to retrieve.

The approach presented in [8] discusses a process component model for process knowledge reuse. Here, the process component model is characterized only using static information (domain, function, performance, lifecycle). We defined a more refined notion of BP knowledge artifacts which can be reused in different stages of the presented modeling lifecycle. In addition, with this approach the user is not able to pose behavioral queries which is a more intuitive way of specifying his requests.

We have not seen other approaches addressing the problem of querying in business process modeling using a rich formal model.

\section{Conclusion}

In this work we have presented an approach to expressive querying for business process artifacts. This approach enables the business expert to have a quick and easy access to the repository of BP artifacts. Our devised approach thus facilitates reuse of existing artifacts and supports ensuring compliance of models to regulations.

We are currently working on a full implementation of the presented approach. As our next step, we aim to advance our approach by supporting more complex cases in behavioral querying. We will investigate the notion of relaxed equivalence between process models, which includes defining similarity measures for

${ }^{2}$ Business Process Management Initiative, http://www.bpmi.org/

${ }^{3}$ Business Process Execution Language, http://www.ibm.com/developerworks/library/specification/ws-bpel/ 
process models with the purpose of quantifying the level of similarity between two models.

\section{Acknowledgments}

This work has been partially supported by the European Union within the FP6 IST project SUPER (FP6-026850).

\section{References}

1. Markovic, I., Pereira, A.C.: Towards a formal framework for reuse in business process modeling. In: Alonso, G., Dadam, P., Rosemann, M. (eds.) BPM 2007. LNCS, vol. 4714. Springer, Heidelberg (2007)

2. Gamma, E., Helm, R., Johnson, R., Vlissides, J.: Design patterns: elements of reusable object-oriented software. Addison-Wesley Longman Publishing Co., Inc., Boston (1995)

3. Hepp, M., Leymann, F., Domingue, J., Wahler, A., Fensel, D.: Semantic business process management: A vision towards using semantic web services for business process management. In: Lau, F.C.M., Lei, H., Meng, X., Wang, M. (eds.) ICEBE, pp. 535-540. IEEE Computer Society, Los Alamitos (2005)

4. ESSI WSML working group: Web Service Modeling Language (WSML) (2006), http://www. wsmo.org/wsml/

5. Milner, R., Parrow, J., Walker, D.: A calculus of mobile processes, i. Inf. Comput. 100(1), 1-40 (1992)

6. BPMI: Business Process Query Language, http://www.service-architecture.com/web-services/articles/ business_process_query_language_bpql.html

7. Beeri, C., Eyal, A., Kamenkovich, S., Milo, T.: Querying business processes. In: Dayal, U., Whang, K.Y., Lomet, D.B., Alonso, G., Lohman, G.M., Kersten, M.L., Cha, S.K., Kim, Y.K. (eds.) VLDB, pp. 343-354. ACM, New York (2006)

8. Mou, Y., Cao, J., sheng Zhang, S.: A process component model for enterprise business knowledge reuse. In: IEEE SCC, pp. 409-412. IEEE Computer Society, Los Alamitos (2004)

\footnotetext{
4 WwW.ip-super.org
} 